\title{
Spinal cord evoked potential monitoring for cervical and thoracic compressive myelopathy
}

\author{
$\mathrm{H} \mathrm{Baba}^{1}$, Y Maezawa ${ }^{1}, \mathrm{~S} \mathrm{Imura}^{1}$, N Kawahara ${ }^{2}$ and K Tomita ${ }^{2}$ \\ ${ }^{I}$ Department of Orthopaedic Surgery, Fukui Medical School, Shimoaizuki 23, Matsuoka, Fukui 910- 11, Japan; \\ ${ }^{2}$ Department of Orthopaedic Surgery, School of Medicine, Kanazawa University, Takaramachi 13-1, Kanazawa 920, \\ Japan
}

\begin{abstract}
Spinal cord evoked potentials (SCEPs) were recorded epidurally in 95 surgical cases of cervical and/or thoracic compressive myelopathy. Abnormal SCEPs occurred in $91 \%$ of the patients with cervical myelopathy and in all with thoracic involvement at the levels suspected to be responsible for neurological damage. Abnormal SCEPs correlated significantly with the severity of spinal cord compromise and symptoms, such as myelopathy. It was not possible to predict postoperative neurological improvement on the basis of the preoperative SCEP findings alone, but better neurological improvement after surgery was closely associated with early recovery of intra- and postoperative SCEPs. Epidural SCEPs are therefore useful in making a level-specific diagnosis, especially in the patient with multilevel vertebral involvement, and in assessing the severity of neurological compromise and surgical outcome to some extent.
\end{abstract}

Keywords: spinal cord evoked potentials; compressive myelopathy; diagnosis; surgical decompression; neurological prognosis

\section{Introduction}

A variety of neurophysiological tests have been used to assess spinal cord function. ${ }^{1-5}$ In assessing the neurological severity in compressive myelopathy and in making a level-specific diagnosis, especially in cases with multiple vertebral involvement in the cervicothoracic region, ${ }^{6-8}$ we have utilized primarily two electrodiagnostic tests: somatosensory cortical evoked potential (SEP) and epidural SCEP recording. Despite the technical problems and their invasiveness, SCEPs can be expected to clearly define the level of neurological compromise necessitating surgical decompression, ${ }^{9-11}$ with the recorded signals being stable electrophysiologically. In this report, we studied the usefulness of epidural SCEP recording in assessing the level(s) of neurological compromise and prognostic value when performing decompressive surgery on patients with cervical and/or thoracic compressive myelopathy.

\section{Patients and methods}

Patients and neurological assessment

Spinal cord evoked potentials were recorded in 95 patients with neurologically and radiologically proven cervical and/or thoracic myelopathy who required surgical decompression at our University Medical

Correspondence: H Baba
Centres. The 58 men and 37 women averaged 47 years of age (range: 27 to 85 years) at the time of admission. Of these, 64 patients had cervical myelopathy, 28 had thoracic involvement, and 3 had profound paresis secondary to a wide range of cervical and thoracic compressive lesions. In accordance with the Ethical Committee Guidelines of the University Medical Centres, informed consent was obtained from all patients included prior to the testing. Forty-one patients agreed to continue testing during the postoperative course, even while awake.

Forty-four patients had cervical spondylotic myelopathy (single-level involvement, 13, multiple level, 31) and 20 had ossification of the posterior longitudinal ligament (OPLL) with multisegmental development in the cervical spine. The surgical approach, either anterior or posterior, or occasionally both, was decided primarily on the basis of findings from myelography and magnetic resonance imaging (MRI). Anterior decompression with fusion was indicated for a patient with a compressive lesion involving at most two vertebrae $(n=16)$. Osteoplastic laminoplasty was used in a patient with a lesion of more than three vertebrae $(n=23)$. Five patients underwent both procedures. All surgery in this series was performed by the senior two authors (HB and/or KT) using a uniform technique.

In 28 patients with thoracic myelopathy, 7 had OPLL, 8 had ossification of the ligamentum flavum, 11 had combined OPLL and ossification of the ligamen- 
tum flavum, and 2 had spondylosis. All patients with thoracic myelopathy had multilevel lesions involving an average of 3.2 vertebrae ( 2 to 8 vertebrae). The decompression area and surgical approach were essentially decided by the radiologic evidence of cord compression anteriorly, posteriorly, or from both directions, similar to an algorithm used in cervical spine disorders.

Preoperative neurological findings and follow-up results were evaluated using the Japanese Orthopaedic Association (JOA) scoring system. ${ }^{2}$ For patients with cervical myelopathy, the postoperative neurological improvement rate was determined by the following equation: (postoperative JOA score - preoperative JOA score)/(17 - preoperative JOA score $) \times 100 \%$. For patients with thoracic myelopathy, excluding assessment categories for upper extremity function (categories 1 and $3 \mathrm{a} ; 6$ points), the improvement rate was obtained similarly to the formula for the cervical spine, with a maximum score of 11 points. The results were assessed as excellent when the improvement rate was $75 \%$ or better, good for $50-74 \%$, fair for $25-$ $49 \%$, and poor for $24 \%$ or worse. Patients were followed-up for an average 4.5 years (range, 1.2 to 7.8 years).

\section{Epidural spinal cord evoked potential recording}

Spinal cord evoked potentials were recorded directly from the dorsal epidural surface near the compressive lesions suspected to be responsible for myelopathy. ${ }^{13-15}$ The recording modality was monopolar throughout the duration of the study. Ascending SCEPs, following stimulation of the lower thoracic spinal cord and descending SCEPs, following stimulation of the cervical cord, were recorded to delineate the range of the cord damage cranially and caudally. The testing was conducted in the awake condition at first, but since 1991, we have performed testing after anaesthetic induction prior to surgery. The testing time was approximately $30 \mathrm{~min}$ at maximum. The stimulating and recording catheter electrodes $(0.75 \mathrm{~mm}$ in diameter, UKG-100-2PMW, Unique Medical, Tokyo, Japan) were introduced at the level just distal to the compressive lesion through epidural needles (French size No. 17 or No. 18) using image intensification. A five-pole recording electrode with 5-mm spacing was used to pick up signals from four different recording sites simultaneously for each test in order to reduce the testing time. The SCEP elicited from an absolutely intact level on imaging studies, near to the lesion, served as a control in each patient. We used DISA 1500 EMG equipment followed by a DISA Evomatic 8000 System (Dantec DISA, Scovlunde, Denmark). However, since 1991, a Medelec ER94 (A Vickers Healthcare, Woking, Surrey, UK) has been used in the operating room without any detectable alterations in the SCEP waveform. The stimulation protocol was as follows: intensity, supramaximal to the visible motor threshold ranging from 35 to 75 volts (4 to $11 \mathrm{~mA}$ ); frequency, $10 \mathrm{~Hz}$; and duration of the rectangularly shaped pulse, $0.2 \mathrm{~ms}$. The recording conditions were set as follows: bandpass, 2 to $2000 \mathrm{~Hz}$; analysis time, 10 to $20 \mathrm{~ms}$; and averaging, 32 to 128 times. A reference electrode was situated between the stimulating and recording electrodes. No complications related to SCEP testing were encountered.

Normal SCEPs, in the present study, consisted of an initial negative spike (N1) followed by a slow negative complex (N2) (Figure 1). The amplitude of N1 measured from the preceding small positive peak ranged 30 to $420 \mu \mathrm{V}(n=17)$, and $\mathrm{N} 2,10$ to $120 \mu \mathrm{V}$ $(n=13)$. Analysing changes seen in the amplitude of the N1 component, the SCEPs were conveniently graded as four subtypes: ${ }^{13,14}$ Type I, normal or decreased amplitude up to $30 \%$ of the control; Type II, amplitude decrement of between $30 \%$ to $50 \%$; Type III, amplitude decrement of more than $50 \%$ or disappearance of the N1 wave; and Type IV, positive waves, i.e., spinal cord evoked injury potentials as designated by Schramm et al, ${ }^{16}$ Halter et al, ${ }^{17}$ and Prestor et al. ${ }^{18}$ Types II, III, and IV are considered abnormal. Spinal cord evoked potentials recorded intraoperatively and serially postoperatively in 41 patients were classified into three groups with respect to the recovery in the amplitude of the SCEPs: group A, immediate recovery showing a Type I response following the decompression maneuver planned preoperatively; group B, SCEP recovery within 2 weeks postoperatively showing a Type I wave or a response improvement of least one grade (e.g., from a Type III to a Type II wave); and group $\mathrm{C}$, no SCEP recovery up to 2 weeks postoperatively or any worsening. We studied the relationship among pre- and postoperative SCEPs, neurologic symptoms, and the neurologic improvement rate after surgery.

\section{Statistical treatment}

SCEP data were collected by two examiners among the authors (NK and YM) and were analysed statistically using an one-way layout ANOVA followed by Scheffe's multiple comparison (Nakayama Books, Tokyo, Japan) with the StatView II Program (Abacus

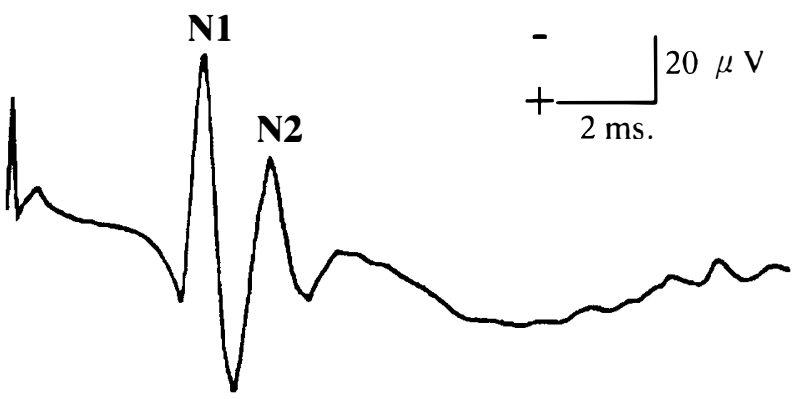

Figure 1 A normal spinal cord evoked potential during ascending recording consisting of two negative $\mathrm{N} 1$ and $\mathrm{N} 2$ waves recorded from the dorsal epidural space. Stimulation was at the T10 level, with monopolar recording at the C5-6 level 
Concepts, Berkeley, CA, USA) on a Macintosh LC630 computer (Apple, Cupertino, CA, USA), with a $P$ value $<0.05$ considered significant.

\section{Results}

Relationship between spinal cord evoked potentials and neurologic severity

In 64 patients with cervical myelopathy, a Type I normal response was observed in 6 , a Type II in 5 , a Type III in 16, and a Type IV in 37. An abnormality was noted $91 \%(58 / 64)$ of the time. All 28 patients with thoracic myelopathy had abnormal SCEPs: a Type II wave was recorded in 6 , a Type III in 7, and a Type IV in 15. All 3 patients with both cervical and thoracic lesions showed abnormal SCEPs (a Type III or Type IV response) in both areas. The relationship between the types of SCEP and the preoperative JOA scores for cervical myelopathy is shown in Figure $2 \mathrm{~A}$ and for thoracic involvement in Figure 2B, and a typical Type

\section{A}

JOA score (point)

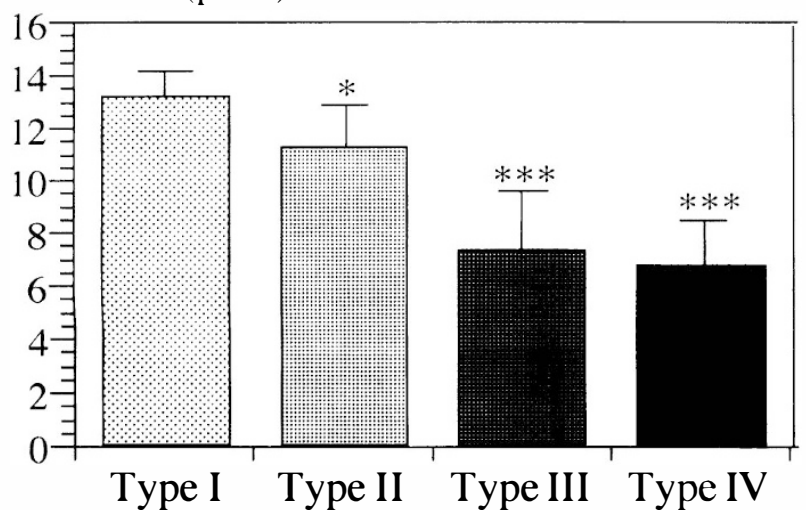

B



Figure 2 The relationship between types of spinal cord evoked potential and preoperative scores. (A) cervical myelopathy $(n=64) ; \quad(B)$ thoracic myelopathy $(n=28)$. $\left({ }^{*}, P<0.05 ; * * *, P<0.001\right)$
A



B



C4-5

C5

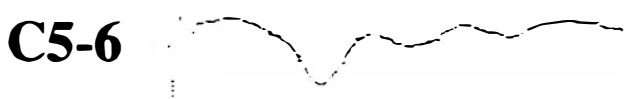

C6

C6-7

C7

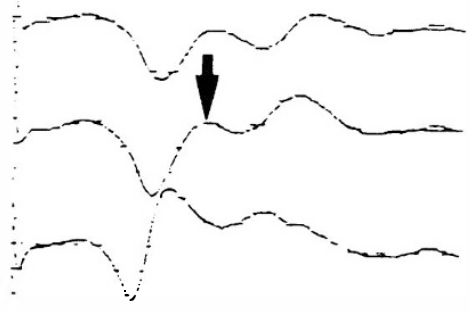

C7-T1

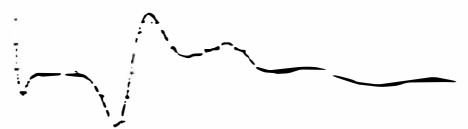

Figure 3 Radiograph (A) and ascending spinal cord evoked potentials (B) of a 65-year-old man with cervical ossified posterior longitudinal ligament. The $\mathrm{N} 1$ component is markedly decreased at the C6-7 level revealing a Type III abnormality (arrow) 


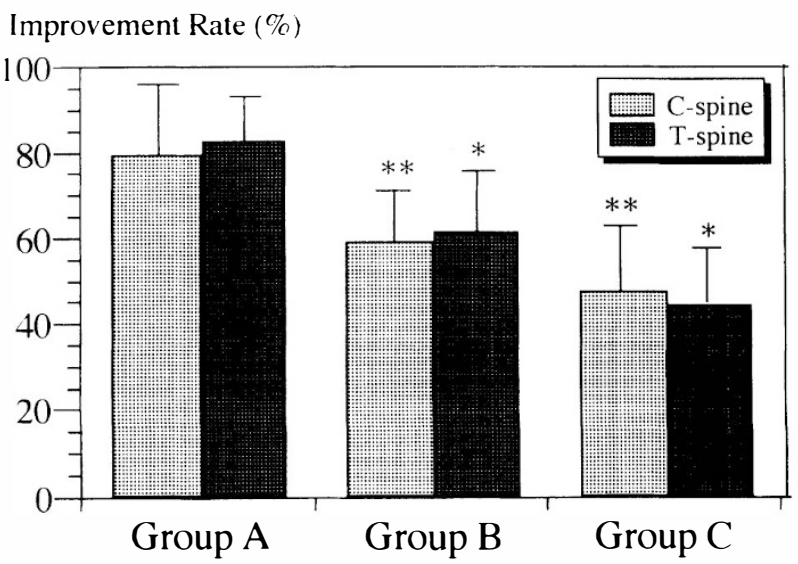

Figure 4 The relationship between recovery of the spinal cord evoked potentials and the postoperative neurologic improvement rate $\left(^{*}, P<0.05 ; * *, P<0.01\right)$

III wave seen in a patient with cervical OPLL myelopathy is illustrated in Figure 3. In patients with cervical myelopathy alone, the mean JOA score in Type I was $13.2 \pm 0.8$ points (mean \pm standard devia- tion); for Type II, $11.3 \pm 1.9$ points; for Type III, 7.4 \pm 2.3 points; and for Type IV, $6.8 \pm 1.7$ points. Patients showing a Type II, III, and IV wave demonstrated significantly lower JOA scores as compared to those with a Type I SCEP. In cases of thoracic myelopathy alone, 6 patients with a Type II response had an average preoperative JOA score of $7.9 \pm 1.1$ points, and 7 with a Type III wave had a significantly lower mean JOA score of $6.5+1.7$ points. The remaining 15 patients with a Type IV abnormality had a significantly lower mean JOA score of $4.5 \pm 1.7$ points preoperatively.

\section{Spinal cord evoked potentials and neurological improvement}

In patients with cervical myelopathy, the 6 with a Type I response achieved an average postoperative neurological improvement rate of $76.3 \pm 18.5 \%$ (mean \pm standard deviation), the 5 with a Type II response an average of $63.2 \pm 24.5 \%$, the 16 with a Type III response an average of $65.3 \pm 19.5 \%$ and the 37 with a Type IV response an average of $59.3 \pm 21.9 \%$. Patients with a Type IV SCEP appeared unlikely to attain favourable neurological improvement, but no statistically significant difference was noted. In cases of

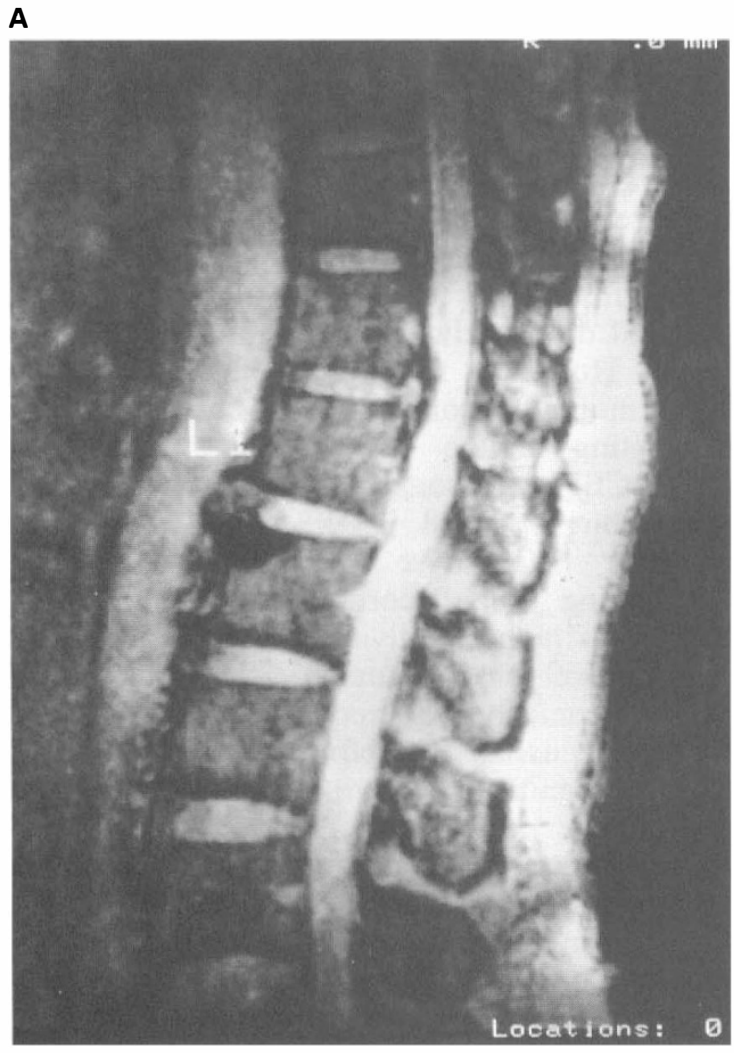

B

(1)

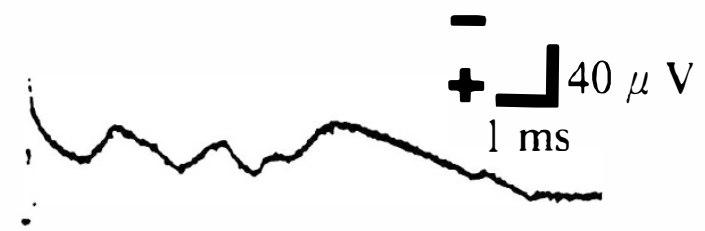

(2)

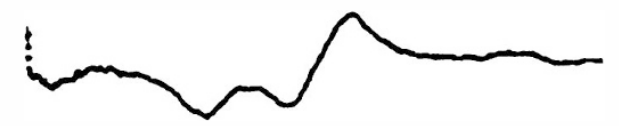

(3)

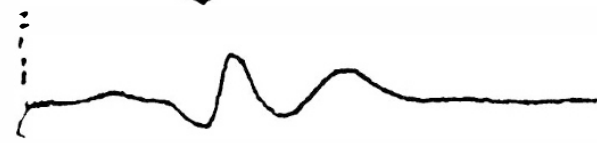

(4)
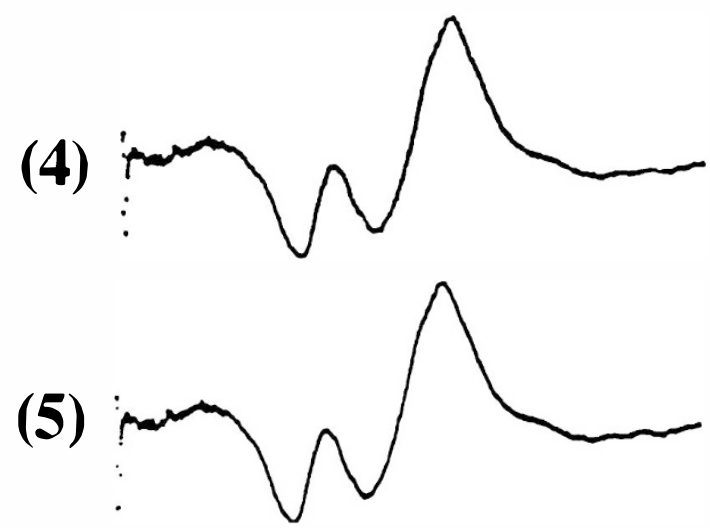

Figure 5 A T2-weighted MRI scan (A) TR, $2000 \mathrm{~ms}$, TE, $80 \mathrm{~ms}$ and spinal cord evoked potentials (B) recorded serially in a patient with thoracic myelopathy due to spondylosis at T11-12 and T12-L1. B: (1): immediately following decompression; (2): 24h later; (3): 2 days later; (4): 8 days later; and (5): 10 days later. The $\mathrm{N} 1$ component is still decreased in the lowest tracing (a Type II response). Stimulation was for a descending evoked response at the T2-3 level with monopolar recording 
thoracic myelopathy, the 6 with a Type II response achieved a postoperative improvement rate of 56.3 $\pm 13.9 \%$, the 7 with a Type III response an average of $49.5 \pm 9.2 \%$, and the 15 with a Type IV response an average of $51.8 \pm 16.3 \%$. No statistically significant differences were observed.

Postoperative neurological improvement correlated significantly with SCEP recovery (Figure 4). In patients with cervical myelopathy, the postoperative improvement rate averaged $79.3 \pm 16.8 \%$ in group A $(n=4), 58.9 \pm 13.3 \%$ in group $\mathrm{B}(n=5)$ and 47.4 $\pm 15.5 \%$ in group C $(n=11)$. Patients in groups B and $\mathrm{C}$ showed significantly less improvement as compared to those in group I. Cases with thoracic myelopathy also demonstrated a statistically significant difference, with an average postoperative improvement rate of $82.3 \pm 11.7 \%$ for group A $(n=5)$, $61.3 \pm 14.4 \%$ for group B $(n=9)$, and $43.9 \pm 13.7 \%$ for group $\mathrm{C}(n=7)$. The one case in group $\mathrm{B}$ in which SCEPs recovered 8 days after thoracic decompression is shown in Figure 5; the patient achieved an improvement of $59 \%$ postoperatively.

\section{Discussion}

In the treatment of patients with multiple level OPLL or spondylosis, it is particularly important to determine the severity of spinal cord compression prior to surgery. For this purpose, epidurally recorded SCEPs have proved helpful in the diagnostic localization of lesions that require decompression. ${ }^{9,11,13,15}$ The authors believe that a localized conduction block secondary to cord compression is well demonstrated by an abnormal wave during epidural SCEP. The important characteristics of SCEPs are as follows: ease of data acquisition, high reproducibility, consistency, and ease in identifying abnormalities (amplitude incrementation and decrementation, and/or latency delay). When utilizing this test, however, it is particularly important to be aware of its technical requirements, the relative advantages over the other recording modalities and, of course, its limitations.

In compressive myelopathy, abnormal SCEPs are frequently elicited at the levels of the damaged spinal cord. $^{9,17}$ In fact, the present study showed that in total, $89(94 \%)$ of 95 patients demonstrated abnormal SCEPs at the compressed levels diagnosed radiologically and neurologically responsible for the myelopathy. For Types III (amplitude decrement of $>50 \%$ of controls) and IV (positive wave SCEPs), these abnormalities occurred in $78(82 \%)$ of all patients tested. In our previous studies, $7,13,14$ we have concluded that the levels at which Type III and IV abnormal responses are recognized should be absolutely included in the decompression, but we are still uncertain whether to include levels with a Type II evoked response. When intending a $\mathrm{C} 3-\mathrm{C} 7$ laminoplasty or a thoracic laminectomy, this uncertainty is not as much of a problem because of the relative ease in extending the decompression, but when anterior surgery is indicated, there can be greater risks than when the decompression is posteriorly performed. ${ }^{19-23}$ Our experimental studies ${ }^{24,25}$ have indicated that the Type II response suggests spinal cord function at a subclinically critical level. Thus, with distinct evidence of a radiological abnormality such as subarachnoidal occlusion in myelograms and MRI signal changes within the spinal cord, the corresponding levels should be included in the area selected for decompression. It should always be appreciated that an apparently mild and longstanding compression with a Type II response may eventually result in severe damage to the spinal cord, leading to Type III and IV abnormalities. To date, however, we have been unable to reach a conclusion regarding inclusion of the levels for which a Type II response is recorded.

Although useful, epidural SCEPs are technically demanding and carry some potential risk for further damaging the already compromised spinal cord. For this reason, Kawahara et $a l^{26}$ have started to utilize non-invasive cortical SEPs following median and ulnar nerve stimulation, to establish the diagnosis. They have suggested that median nerve-stimulated SEPs reflect the neurological pathology responsible at the C4-5 level and ulnar nerve-stimulated SEPs at the C6-7 level. We agree with the idea that an invasive test such as SCEP recording is replaced with the noninvasive method of cortical SEPs. The present study was originally aimed primarily for determination of the compromised spinal cord level(s), and the postoperative SCEP monitoring was by no means the necessary technique. For postoperative neurological assessment, the SEPs monitoring technique is absolutely a recommended choice with regard to noninvasiveness and electrophysiological stability in recording the evoked responses. Single- and two-level involvement do not always require invasive testing for diagnosis. However, for lesions suspected at three levels or more, or compressive lesions non-contiguously existing in the cervical and thoracic spine, epidural SCEPs can suggest not only a level-specific diagnosis, but also aid in the selection of surgical procedure. ${ }^{11,27}$ We believe MRI offers precise information regarding spinal cord compression, however, because of its high sensitivity in imaging, a clinically 'innocent' level not actually responsible for the neurologic compromise may be included in the decompressed areas. Therefore, we emphasize that the clinical significance of the SCEP testing lies in confirming the level-specific diagnosis and it can modify the choice of surgical procedures initially planned based upon the neurological examination and the imaging studies alone.

The present study demonstrates that preoperative neurological scores correlate with epidural SCEP abnormalities. Also, the degree of spinal cord functional compromise is related to the type of SCEP. However, we were unable to predict postoperative neurological improvement on the basis of preoperative SCEP findings alone. Interestingly, a 
Type III or even a Type IV response did not necessarily predict an unfavourable surgical outcome. Patients with a Type III or a Type IV SCEP response preoperatively showed a trend for less improvement postoperatively, but not to a statistically significant degree. To date, we are unable to give any valid explanation as to why the preoperative SCEPs would not predict postoperative neurologic improvement. Shinomiya et $a l^{28}$ have reported similar results, but they have suggested that epidural SCEP abnormalities occurring in multilevel lesions may imply an unfavourable neurological outcome, as compared to a singlelevel lesion. One possible explanation may involve limitations in obtaining preoperative SCEPs. Although some motor impulses are transmitted in descending recordings, SCEPs using the present technique mainly reflect sensory transmission within the spinal cord. Using a weightdrop technique applied dorsally to the cat spinal cord, we have found that SCEPs from the posterior epidural space do not accurately reflect all the injurious events occurring in the anterior and anterolateral portion of the spinal cord. ${ }^{25,29}$ The N1 component of the SCEP originates mainly in the larger fibres running predominantly in the lateral and posterolateral columns and in pathways without interneuronal activity. Thus, neurological injury to the motor tracts within the anterolateral portion of the spinal cord may be missed during SCEP recording. As Takano et $a l^{30}$ also have suggested, this may explain the difficulty in predicting the neurological outcome preoperatively. From this point of view, the combined use of motor evoked potential ${ }^{31 \cdots 34}$ recording, either by transcranial or spinal cord stimulation, may offset limitations of epidural SCEPs.

Interestingly, postoperative neurological improvement correlated significantly with SCEP recovery intraoperatively and early following surgery. The improvement in neural transmission following decompression may contribute to the SCEP recovery. Recovery in the SCEPs, mainly in their amplitude and waveform configuration, highlights the appropriateness of the surgical approach and decompression. It may be safely stated that early recovery in SCEPs predicts a better neurologic improvement following surgery. Furthermore, although we have never encountered a serious problem, SCEPs may be of value in reducing the risk of iatrogenic injury to the spinal cord during decompression. Anterior resection of large spondylotic spurs and ossified posterior longitudinal ligaments may carry potential risks for the spinal cord to be further damaged, especially when resecting the lesion anteriorly en bloc. ${ }^{20,27}$ If the SCEPs rapidly deteriorate during the decompressive manipulation, the surgical maneuver should be stopped immediately. However, a Type IV response cannot be monitored intraoperatively. On the other hand, cortical SEP is expected to be a better alternative for monitoring neurologic function postoperatively. The recording of SEPs is non-invasive and easily reproducible with considerable sensitivity in assessing cord function. We have previously reported that the P11, P14, and N18 spike of median nerve SEPs have recovered well in relation to the degree of neurological improvement in patients who have undergone decompression surgeries for cervical OPLL myelopathy. ${ }^{6,35}$ Latency and amplitude recoveries in these spikes during the early postoperative period, within 2 months of surgery, were later found to be prognostic signs of neurological improvement. This was similar to the experience of SCEPs. Each spike of an SEP is considerably smaller in amplitude than those of SCEPs and is sometimes difficult to be recognized during surgery. Therefore, an effort must be made to minimize the SEP artifact and to increase the electrophysiological stability as well as sensitivity. Postoperative monitoring using SCEPs may be useful as shown in the current study but, from a practical point of view, the SCEP monitoring technique is limited for a patient having multiple compressive lesions who should be examined for establishing the level-specific diagnosis and have postoperative neurological monitoring by cortical SEP recording.

\section{Conclusions}

The current study indicates that epidural SCEPs are beneficial in making a level-specific diagnosis, especially in patients with multilevel vertebral involvement. The preoperative SCEPs are less useful in predicting neurological prognosis, but to some extent, the early recovery of SCEPs predicts a more favourable surgical outcome.

\section{References}

1 Brown RH, Nash CL Jr. Intra-operative spinal cord monitoring. Chapter 27. In: Frymoyer JW (ed). The Adult Spine: Principles and Practice. Raven Press: New York 1991, pp 557-558.

2 Dawson EG, Sherman JE, Kanim LEA, Nuwer MR. Spinal cord monitoring: results of the Scoliosis Research Society and European Spinal Deformity Society survey. Spine 1991; 16: S361-S365.

3 Kalkman CJ, Drummond JC. Intraoperative evaluation using somatosensory and motor evoked potentials. In: Garfin SR, Northrup BE (eds). Surgery for Spinal Cord Injuries. Raven Press: New York 1993, pp 33-44.

4 Nash CL Jr, Brown RH. Current concepts review: spinal cord monitoring. J Bone Joint Surg Am 1989; 71: 627-630.

5 Zentner J, Rieder G. Diagnostic significance of motor evoked potentials in space-occupying lesions of the brainstem and spinal cord. Eur Arch Psychiatr Neurol Sci. 1990; 239: 285-289.

6 Baba H, Shima I, Tomita K, Umeda S, Sawada Y. Somatosensory evoked potentials in cervical myelopathy. In: Schramm J, Jones SJ (eds). Spinal Cord Monitoring. Springer-Verlag: Berlin Heidelberg 1985, pp 284-289.

7 Baba H, Tomita K, Umeda S. Clinical study of spinal cord evoked potentials. In: Ducker TB, Brown RH (eds). Neurophysiology and Standards of Spinal Cord Monitoring. SpringerVerlag: New York 1988, pp 216-221.

8 Baba $\mathrm{H}$, Tomita $\mathrm{K}$, Maezawa $\mathrm{Y}$, Imura $\mathrm{S}$. Intermittent claudication of the spinal cord due to ossification of the ligamentum flavum. Int Orthop 1993; 17: 169-172. 
25 Baba H, Kawahara N, Nagata S, Tomita K, Imura S. Spinal somatosensory evoked potentials in compressive and distraction injury. Paper presented at the 59th Annual Meeting of the American Academy of Orthopaedic Surgeons, February 20-25, 1992, Washington, D.C., USA.

26 Kawahara N, Baba H, Nagata S, Kikuchi Y, Tomita K, Yugami $\mathrm{H}$. Level diagnosis of cervical myelopathy using somatosensory evoked potentials. In: Jones SJ, Boyd S, Hetreed M, Smith NJ (eds). Handbook of Spinal Cord Monitoring. Kluwer Academic: Dordrecht 1994, pp $192-196$.

27 Tomita K, Kawahara N, Baba H, Kikuchi Y, Nishimura H. Circumspinal decompression for thoracic myelopathy due to ossification of the posterior ligament and ligamentum flavum. Spine 1990; 15: 1114- 1120.

28 Shinomiya K, Okamoto A, Komori H, Matsuoka T, Yoshida $\mathrm{H}$, Muto N, Furuya K. Prognosticating study for cervical myelopathy using evoked spinal cord potentials. Spine 1990; 15: $1053-1057$

29 Baba H, Tomita K, Umeda S, Kawahara N, Nagata S, Nomura $\mathrm{S}$, Yugami H. Experimental ascending evoked potentials in spinal cord injury. In: Ducker TB, Brown RH (eds). Neurophysiology and Standards of Spinal Cord Monitoring. Springer-Verlag: New York 1988, pp 46-51.

30 Takano H, Kitagawa H, Yamamoto N, Takakuwa K, Tsuji H. Origins and conducting tracts of evoked spinal cord potentials in cats. J Spinal Disord 1991; 4: 455-461.

31 Barker AT, Freeston IL, Jalinous R, Jarratt JA. Noninvasive stimulation of motor pathways within the brain using timevarying magnetic field. Electroencephalogr Clin Neurophysiol 1985; 61: $245-246$

32 Dimitrijevic MR, Eaton WJ, Sherwood AM, Van der Linden C. Assessment of corticospinal tract integrity in human chronic spinal cord injury. In: Rossini PM, Marsden CD (eds). Neurology and Neurobiology, Volume 41, Non-invasive Stimulation of Brain and Spinal Cord. Fundamentals and Clinical Applications. Alan R. Liss: New York 1988, pp 243-253.

33 Dvorák J, Herdmann J, Janssen B, Theiler R, Grob D. Motorevoked potentials in patients with cervical spine disorders. Spine 1990; 15: $1013 \cdots 1016$.

34 Katz RT, Vandenburg C, Weinberge D, Cadwell J. Magnetoelectric stimulation of human motor cortex: normal values and potential safety issues in spinal cord injury. Arch Phys Med Rehabil 1990; 71: 597-600.

35 Baba H, Tomita K, Umeda S, Kawahara N, Nagata S, Kikuchi Y. Somatosensory cortical evoked potentials in cervical OPLL myelopathy. Clin Orthop Surg (Tokyo) 1988; 23: 1409-1416. 